\title{
Efficient Artificial Light-Harvesting System Based on Supramolecular Peptide Nanotubes in Water
}

\author{
Qiao Song, Sofia Goia, Jie Yang, Stephen C. L. Hall, Michael Staniforth, Vasilios G. Stavros, \\ and Sébastien Perrier*
}

Cite This: J. Am. Chem. Soc. 2021, 143, 382-389

Read Online

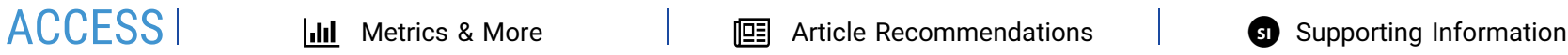

ABSTRACT: Artificial light-harvesting systems in aqueous media which mimic nature are of significant importance; however, they are often restrained by the solubility and the undesired aggregation-caused quenching effect of the hydrophobic chromophores. Here, we report a generalized strategy toward the construction of efficient artificial lightharvesting systems based on supramolecular peptide nanotubes in water. By molecularly aligning the hydrophobic chromophores along the nanotubes in a slipped manner, an artificial light-harvesting system with a two-step sequential Förster resonance energy transfer process is successfully fabricated, showing an energy transfer efficiency up to $95 \%$ and a remarkably high fluorescence quantum yield of $30 \%$, along

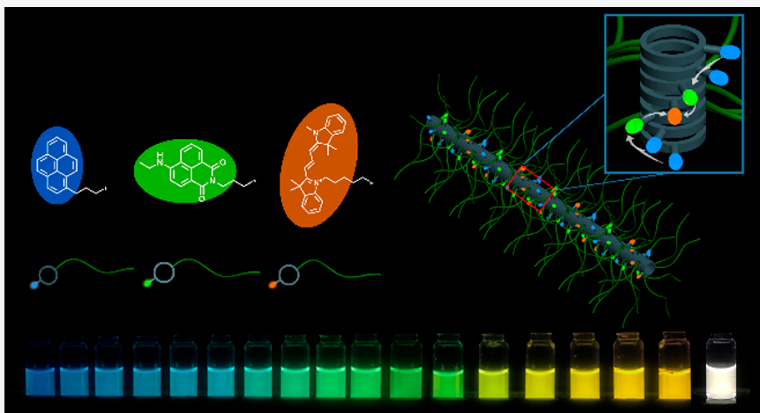
with high stability. Furthermore, the spectral emission could be continuously tuned from blue through green to orange, as well as outputted as a white light continuum with a fluorescence quantum yield of $29.9 \%$. Our findings provide a versatile approach of designing efficient artificial light-harvesting systems and constructing highly emissive organic materials in aqueous media.

\section{INTRODUCTION}

In nature, plants and photosynthetic bacteria are able to capture, transfer, and store solar energy utilizing their highly efficient light-harvesting systems. ${ }^{1,2}$ Within these light-harvesting systems, chromophores are aligned into arrays by the surrounding proteins, which are capable of absorbing and transferring light energy from one to another, until the energy is funneled to the reaction center. ${ }^{3-5}$ By mimicking nature, artificial systems have been developed to achieve efficient harvesting of light energy through the Förster resonance energy transfer (FRET) processes from donor chromophores to acceptor chromophores, ${ }^{6-10}$ including self-assembled structures such as dendrimers, ${ }^{11,12}$ supramolecular polymers, ${ }^{13-16}$ gels, ${ }^{17,18}$ micelles, ${ }^{19}$ vesicles, ${ }^{20-23}$ and bioinspired structures. $^{24-27}$ However, limited by the solubility and the undesired aggregation-caused quenching (ACQ) effect of the hydrophobic chromophores in water, light-harvesting systems built in aqueous environments often show unsatisfying performance with considerably low fluorescence quantum yields. Within this limitation, while the utilization of fluorophores with aggregation induced emission (AIE) properties has been shown to be a powerful tool for constructing light-harvesting systems with relatively high quantum yields, ${ }^{28-35}$ a generalized strategy is essential to tackle this issue. Moreover, artificial light-harvesting systems with high stability are of significant importance for practical applications, which is rarely emphasized in supramolecular systems.
Assemblies of cyclic peptide-polymer conjugates are a relatively new type of supramolecular polymers. ${ }^{36-40}$ The alternating D- and L-amino acid configuration of the cyclic octapeptides enables strong multiple hydrogen bonding interactions between the flat rings of the cyclic peptides, driving the formation of nanotubular assemblies. ${ }^{41-43}$ Conjugating hydrophilic polymers onto cyclic peptides prevents their lateral aggregation and improves their solubility and stability in aqueous environments, forming self-assembled polymeric nanotubes with well-defined structure. ${ }^{44-46}$ Inspired by the highly ordered structures guided by cyclic peptides, we envisioned that by attaching the hydrophobic chromophores onto the cyclic peptide-polymer conjugates, they could be molecularly aligned along the supramolecular polymeric nanotubes in a slipped way, enabling us to design efficient artificial light-harvesting systems while avoiding the undesired ACQ effect of the chromophores in water. Moreover, due to the strong binding affinity between the cyclic peptides, the artificial light-harvesting system is expected to exhibit very good stability.

Received: October 20, 2020

Published: December 22, 2020 
Scheme 1. Artificial Light-Harvesting System Based on Supramolecular Peptide Nanotubes in Water: (A) Chemical Structures of three Fluorophore-Cyclic Peptide-Polymer Conjugates; and (B) Cartoon Illustration of the Artificial Light-Harvesting System

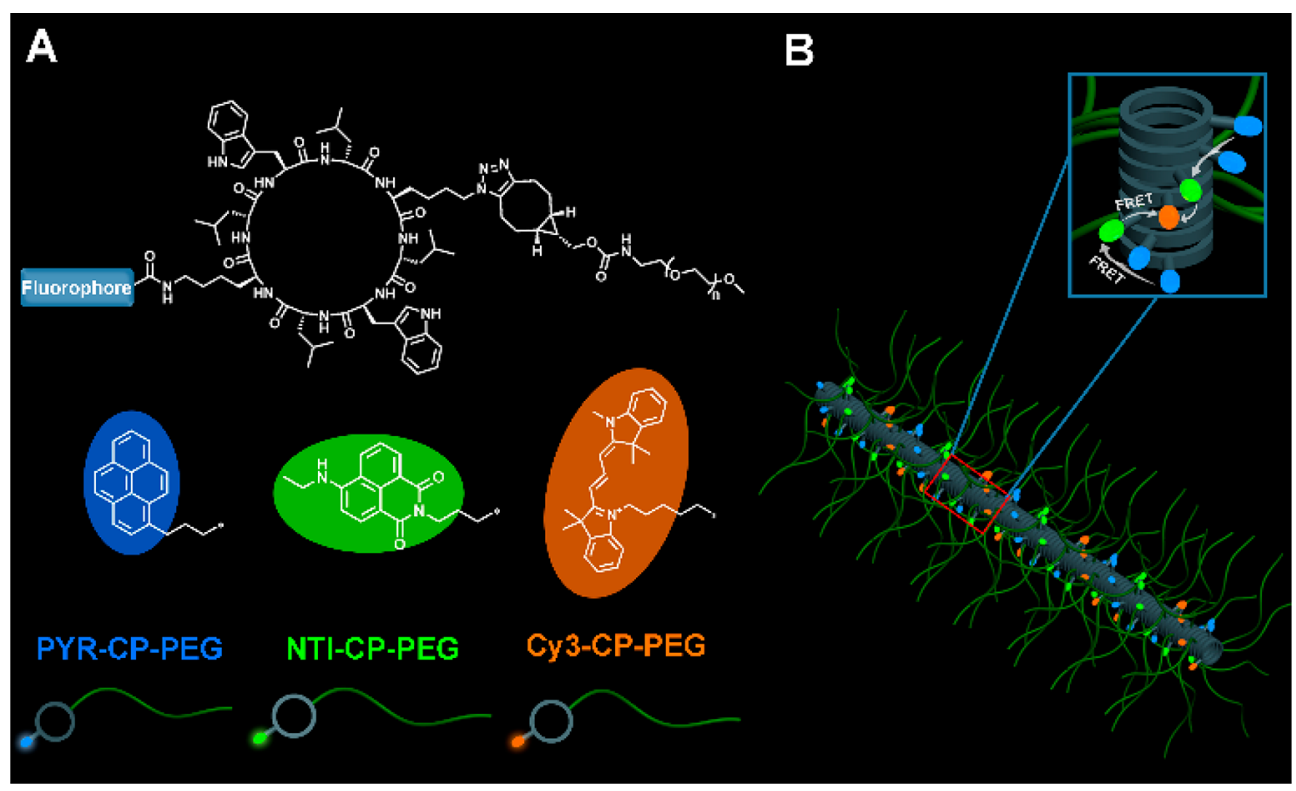

In this work, we report an efficient artificial light-harvesting system with a two-step sequential FRET process constructed by molecularly aligning the hydrophobic chromophores along the self-assembled peptide nanotubes in water. As shown in Scheme 1, three fluorophore-cyclic peptide-polymer conjugates, pyrene-cyclic peptide-poly(ethylene glycol) (PYR-CPPEG), naphthalene monoimide-cyclic peptide-poly(ethylene glycol) (NTI-CP-PEG), and cyanine3-cyclic peptide-poly(ethylene glycol) (Cy3-CP-PEG), are designed and synthesized. An efficient FRET process takes place from PYR-CPPEG to NTI-CP-PEG when coassembling both into supramolecular peptide nanotubes in water, which meets the requirement of promising candidates for light-harvesting systems. $^{7}$ A two-step sequential FRET process could be further realized by incorporating a third conjugate, Cy3-CPPEG. In this regard, light energy is transferred from PYR-CPPEG to NTI-CP-PEG, and subsequently transferred to Cy3CP-PEG. More importantly, due to the slipped stacking arrangement of the fluorophores along the supramolecular peptide nanotubes, the typical ACQ effect is largely inhibited with fluorescence quantum yields measured to be over $30 \%$. Moreover, the emission color could be continuously tuned from blue to green and finally to orange simply by adjusting the ratio of the three conjugates. Particularly, a pure white-light emission of $(0.32,0.34)$ in CIE 1931 coordinates is obtained at a specific donor/acceptor ratio with a fluorescence quantum yield as high as $29.9 \%$. The supramolecular peptide nanotubebased system does not only perform as a highly efficient artificial light harvesting system in aqueous media, but also provides a versatile methodology toward constructing lightemitting materials with high fluorescence quantum yields.

\section{RESULTS AND DISCUSSION}

Design, Synthesis, and Self-Assembly of Fluorophore-Cyclic Peptide-Polymer Conjugates. The three fluorophore-cyclic peptide-polymer conjugates, PYR-CP-PEG, NTI-CP-PEG, and Cy3-CP-PEG, were synthesized by attaching the corresponding fluorophores via either NHS coupling chemistry or HATU coupling chemistry, followed by conjugating the hydrophilic polymer PEG $\left(M_{\mathrm{n}}=5000 \mathrm{~g}\right.$ $\mathrm{mol}^{-1}$ ) via strained alkyne/azide group ligation. PYR-CP-PEG, NTI-CP-PEG, and Cy3-CP-PEG were subsequently characterized by ESI-MS, HPLC, and GPC (Figures S1-S7 of the Supporting Information, SI). Two fluorophore-polymer conjugates in the absence of cyclic peptides, PYR-PEG and NTI-PEG, were also synthesized as control compounds (Figures S8-S10).

Multiple hydrogen bonding interactions between the cyclic peptides are the driving forces of forming self-assembled polymeric nanotubes. The self-assembling behavior of conjugates PYR-CP-PEG, NTI-CP-PEG, and Cy3-CP-PEG in aqueous solution was investigated by small angle neutron scattering (SANS) and transmission electron microscopy (TEM). Figure 1A shows the reduced, corrected scattering data for PYR-CP-PEG, NTI-CP-PEG, and Cy3-CP-PEG conjugates in water. Using SASfit software, the data could be fitted with a cylindrical micelle model, suggesting the selfassemblies forming polymer nanotubes in aqueous solutions. ${ }^{47}$ Subtle structural differences were observed between nanotubes formed by each conjugate, hypothesized to be induced by the physicochemical properties of different fluorophores (SI Table S1). These conjugates could also be visualized by TEM (Figures 1B,C and S11), showing 1D structures for the three conjugates in aqueous media. The diameter of the nanotubes was narrowly distributed around $8 \mathrm{~nm}$, while the length varied within $100 \mathrm{~nm}$, which is consistent with the proposed selfassembled polymeric nanotube structures. Furthermore, similar structures were also observed by SANS and TEM for the mixtures of these three conjugates (Figures 1D and S12).

Proof and Evaluation of the Artificial Light-Harvesting System with a Two-Step Sequential FRET Process. Two factors are required for an efficient FRET system: (1) the emission spectrum of the donor fluorophore must overlay well with the absorption spectrum of the acceptor fluorophore; and (2) the donor and acceptor should be close to each other (within $\sim 10 \mathrm{~nm}$ ). ${ }^{48,49}$ We initially investigated the FRET 
A

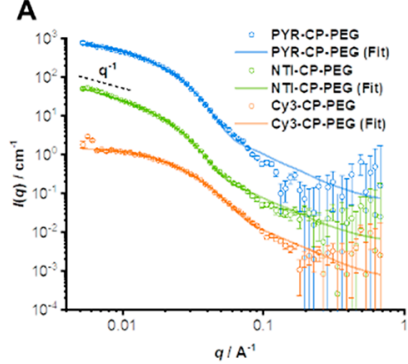

C

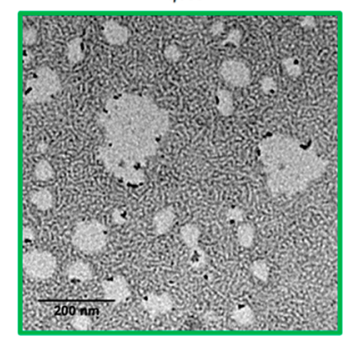

B

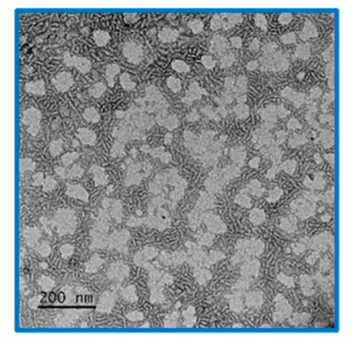

D

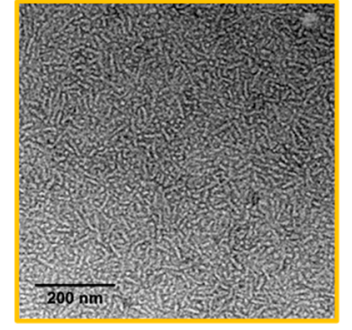

Figure 1. Characterization of self-assembled peptide nanotubes. SANS scattering data and fitting to a cylindrical micelle model of PYR-CP-PEG, NTI-CP-PEG, and Cy3-CP-PEG (A); TEM images of PYR-CP-PEG (B); NTI-CP-PEG (C); and a mixture of three conjugates (D).

process between PYR-CP-PEG and NTI-CP-PEG by measuring the absorption and emission spectra of PYR-CP-PEG and NTI-CP-PEG as well as the two control compounds PYR-PEG and NTI-PEG. Interestingly, the emission spectrum of PYRPEG showed minimal overlap with the absorption spectrum of NTI-PEG (Figure S13), in contrast to the emission spectrum of PYR-CP-PEG which overlapped well with the absorption spectrum of NTI-CP-PEG (Figure 2A). This could be ascribed to the formation of pyrene excimers caused by the stacking of the cyclic peptides, as indicated by the peak in the emission spectrum at $460 \mathrm{~nm}$. As a result, an efficient energy transfer process is expected to occur when coassembling PYR-CP-PEG
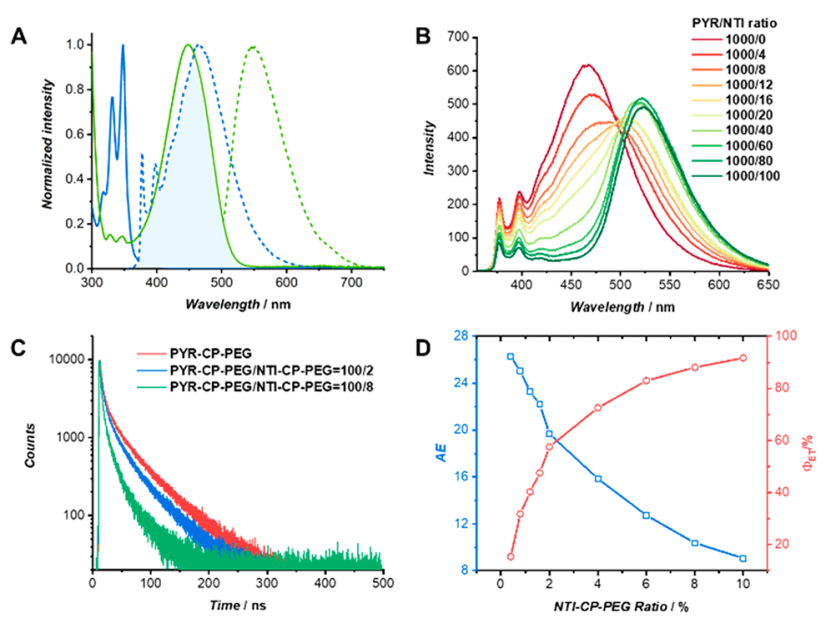

Figure 2. FRET process between PYR-CP-PEG and NTI-CP-PEG. (A) Normalized fluorescence spectra (dashed curves) of PYR-CPPEG (blue trace, $\lambda_{\mathrm{ex}}=335 \mathrm{~nm}$ ) and NTI-CP-PEG (green trace, $\lambda_{\mathrm{ex}}=$ $460 \mathrm{~nm}$ ), and their normalized absorbance spectra (solid curves). (B) Fluorescence spectra of PYR-CP-PEG in water with different concentrations of NTI-CP-PEG $\left(\lambda_{\mathrm{ex}}=335 \mathrm{~nm}\right)$. (C) Fluorescence decay profiles of PYR-CP-PEG and PYR-CP-PEG/NTI-CP-PEG. (D) $\mathrm{AE}$ and $\Phi_{\mathrm{ET}}$ at different NTI-CP-PEG/PYR-CP-PEG ratios.

and NTI-CP-PEG conjugates. Indeed, as demonstrated in Figure 2B, with the increase of NTI-CP-PEG/PYR-CP-PEG ratio, PYR excimer emission intensity at $460 \mathrm{~nm}$ decreased gradually, and the emission intensity of the NTI fluorophore at $520 \mathrm{~nm}$ increased when excited at $335 \mathrm{~nm}$. Fluorescence decay experiments were performed to further confirm the energy transfer process. As shown in Figure 2C, the fluorescence decay of PYR-CP-PEG was significantly faster in the presence of NTI-CP-PEG. The decay curves were fitted using a threeexponential decay model (Figure S14, Table S2), showing the fluorescence lifetimes of $\tau_{1}=3.4 \mathrm{~ns}, \tau_{2}=20.9 \mathrm{~ns}$, and $\tau_{3}=67.2$ $\mathrm{ns}$ for PYR-CP-PEG. The fluorescence lifetimes decreased to $\tau_{1}=2.7 \mathrm{~ns}, \tau_{2}=16.7 \mathrm{~ns}$, and $\tau_{3}=59.4 \mathrm{~ns}$ when mixing with $2 \%$ NTI-CP-PEG, and further decreased to $\tau_{1}=1.5 \mathrm{~ns}, \tau_{2}=9.8 \mathrm{~ns}$, and $\tau_{3}=40.9 \mathrm{~ns}$ by coassembling $8 \%$ NTI-CP-PEG. This clearly indicates that the energy can be transferred from PYRCP-PEG to NTI-CP-PEG when coassembling both into supramolecular polymeric nanotubes. To prove the importance of forming self-assembled polymeric nanotubes, control experiments were conducted. As shown in Figure S15, no FRET was observed when mixing either PYR-PEG/NTI-CPPEG or PYR-CP-PEG/NTI-PEG in aqueous solutions, suggesting that the closely packed structure formed by coassembling both fluorophores into polymeric nanotubes is essential for FRET.

To quantitatively evaluate its performance as an artificial light-harvesting system, energy transfer efficiency $\left(\Phi_{\mathrm{ET}}\right)$ and antenna effect (AE) were measured and calculated at different PYR-CP-PEG/NTI-CP-PEG ratios (Figure 2D, Table S3). $\Phi_{\mathrm{ET}}$ represents the fraction of absorbed energy by the donor that is transferred to the acceptor, while AE describes how much brighter the acceptor emits by exciting the donor instead of directly exciting the acceptor. Overall, the $\Phi_{\mathrm{ET}}$ values increased with the increase of NTI-CP-PEG ratio and eventually reached up to $90 \%$. The $\mathrm{AE}$ values, however, decreased with the increase of NTI-CP-PEG ratio. Particularly, we found that the $\mathrm{AE}$ value could be maintained above 10 while the $\Phi_{\mathrm{ET}}$ reached $90 \%$. For example, at a PYR-CP-PEG/ NTI-CP-PEG ratio of $100 / 4, \Phi_{\mathrm{ET}}$ is calculated to be $72.6 \%$ with an AE value of 15.8; at a PYR-CP-PEG/NTI-CP-PEG ratio of $100 / 8, \Phi_{\mathrm{ET}}$ is $88.1 \%$ with an $\mathrm{AE}$ value of 10.4 . More importantly, PYR-CP-PEG exhibited a fluorescence quantum yield as high as $39.7 \pm 3.7 \%$, while the quantum yield of PYRCP-PEG/NTI-CP-PEG $=100 / 8$ was measured to be $34.1 \pm 1.5 \%$. To the best of our knowledge, this represents one of the highest values reported for aqueous artificial lightharvesting systems, ${ }^{22,23,31,35}$ which is attributed to both the slipped stacking arrangement of the fluorophores along the supramolecular peptide nanotubes and the highly efficient energy transfer process.

An ideal artificial light-harvesting system should be robust in various environmental conditions. Due to the high fidelity of the multiple hydrogen bonding interactions between the cyclic peptides, this supramolecular peptide nanotube-based lightharvesting system exhibits impressive stability at a wide range of concentrations and temperatures. Herein, $\mathrm{AE}$ and $\Phi_{\mathrm{ET}}$ were used to evaluate the performance of the light-harvesting system (PYR-CP-PEG/NTI-CP-PEG = 100/8) while varying the concentration or temperature. When decreasing the concentration from $50 \mu \mathrm{M}$ to as low as $0.39 \mu \mathrm{M}$ while maintaining the same chromophore ratio, $\Phi_{\mathrm{ET}}$ remained constant while $\mathrm{AE}$ showed an increasing trend, suggesting its high stability upon dilution (Figure S16). Furthermore, as shown in Figure S17, 
AE and $\Phi_{\mathrm{ET}}$ were both decreasing slowly with the increase of temperature. Nevertheless, even at $80{ }^{\circ} \mathrm{C}$, only less than $10 \%$ decrease of $\mathrm{AE}$ and $\Phi_{\mathrm{ET}}(4.7 \%$ and $7.1 \%$, respectively) was observed. Hence, the stability of this supramolecular peptide nanotube-based system demonstrates its great potential for practical applications as an artificial light-harvesting system.

Nature uses multistep sequential energy transfer rather than only one-step energy transfer to make better utilization of light in a wide wavelength range. We explored the possibility of fabricating multistep sequential energy transfer system based on self-assembled polymeric nanotubes. Cy3-CP-PEG conjugate was rationally chosen as the second acceptor to harvest light emitted from NTI-CP-PEG, as the absorption spectrum of Cy3-CP-PEG overlaps well with the emission spectrum of NTI-CP-PEG (Figure 3A). In this respect, it is anticipated that
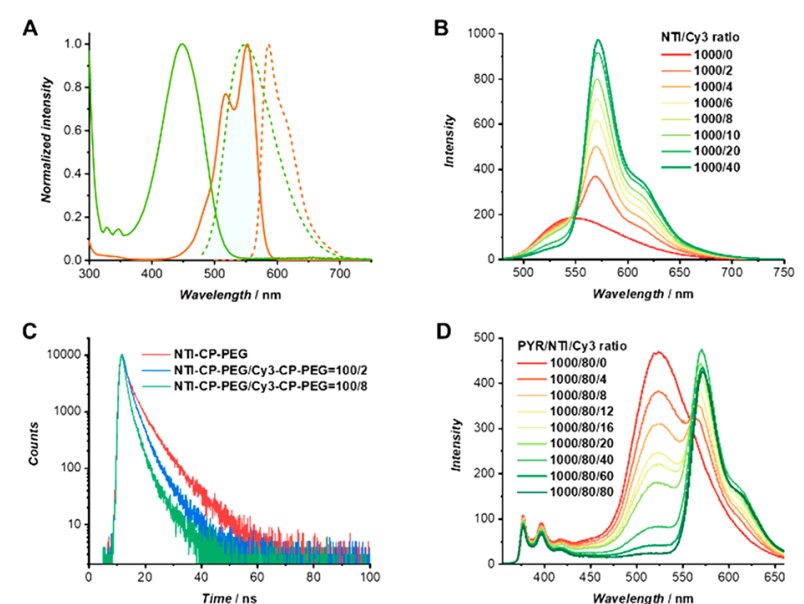

Figure 3. Two-step sequential FRET process between PYR-CP-PEG, NTI-CP-PEG, and Cy3-CP-PEG. (A) Normalized fluorescence spectra (dashed curves) of NTI-CP-PEG (green trace, $\lambda_{\mathrm{ex}}=460$ $\mathrm{nm}$ ) and Cy3-CP-PEG (orange trace, $\lambda_{\mathrm{ex}}=476 \mathrm{~nm}$ ), and their normalized absorbance spectra (solid curves). (B) Fluorescence spectra of NTI-CP-PEG in water with the addition of different molar ratios of Cy3-CP-PEG $\left(\lambda_{\mathrm{ex}}=460 \mathrm{~nm}\right)$. (C) Fluorescence decay profiles of NTI-CP-PEG and NTI-CP-PEG/Cy3-CP-PEG. (D) Fluorescence spectra of PYR-CP-PEG/NTI-CP-PEG $=100 / 8$ in water with the addition of different molar ratios of Cy3-CP-PEG $\left(\lambda_{\text {ex }}\right.$ $=335 \mathrm{~nm}$ ).

NTI-CP-PEG could act as a bridge to harvest light emitted by PYR-CP-PEG, and subsequently transfer the energy to Cy3CP-PEG. First, the FRET efficiency between NTI-CP-PEG and Cy3-CP-PEG was studied. As evidently illustrated by Figure 3B, with the increase of Cy3-CP-PEG concentration, NTI emission intensity at $550 \mathrm{~nm}$ decreased gradually, and the emission intensity of $\mathrm{Cy} 3$ at $570 \mathrm{~nm}$ increased significantly when excited at $460 \mathrm{~nm}$. Fluorescence decay experiments were also conducted to confirm the FRET process between NTICP-PEG and Cy3-CP-PEG, which shows a faster fluorescence decay of NTI-CP-PEG in the presence of Cy3-CP-PEG (Figures 3C and S18, and Table S4). Second, to facilitate the proposed sequential energy transfer process, a threecomponent system was constructed by coassembling PYRCP-PEG, NTI-CP-PEG and Cy3-CP-PEG. As shown in Figure 3D, when Cy3-CP-PEG was added into PYR-CP-PEG/NTICP-PEG, the emission band ascribed to NTI decreased and a new emission band at $570 \mathrm{~nm}$ belonging to Cy3-CP-PEG appeared simultaneously when excited at $335 \mathrm{~nm}$. These data indicate the occurrence of a two-step sequential FRET process, which is further supported by fluorescence decay experiments (Figure S19, Table S5). It should be noted that the energy might be transferred directly from PYR-CP-PEG to Cy3-CPPEG; however, we anticipate this to be minor owing to the poor overlap between the emission spectrum of PYR-CP-PEG and the absorption spectrum of Cy3-CP-PEG (Figure S20). Thus, in the three-component system, a weak one-step energy transfer from PYR-CP-PEG to Cy3-CP-PEG is possible, though, when excited at $335 \mathrm{~nm}$, a two-step sequential FRET process is anticipated to be preferred. Similar to the two-component system composed of PYR-CP-PEG and NTI$\mathrm{CP}-\mathrm{PEG}$, increasing the relative concentration of the third component, Cy3-CP-PEG, caused a significant increase in $\Phi_{\mathrm{ET}}$, reaching $95.0 \%$ at a low donor/acceptor ratio of $100 / 8 / 8$ (Figure S21, Table S6). The fluorescence quantum yield was determined to be $29.9 \pm 0.7 \%$ at a ratio of $100 / 8 / 2$, further emphasizing the high energy transfer efficiency between the three components. The above results confirm that the supramolecular polymeric nanotubes fabricated by PYR-CPPEG, NTI-CP-PEG, and Cy3-CP-PEG could function as a promising artificial light-harvesting system with high energy transfer efficiency and high fluorescence quantum yield.

To further understand the dynamical processes involved in the energy transfer between the fluorophores, transient electronic absorption spectroscopy (TEAS) measurements were performed on PYR-CP-PEG, NTI-CP-PEG, and Cy3CP-PEG conjugates. Transient absorption spectra (TAS) were collated following photoexcitation at $335 \mathrm{~nm}$ of the aqueous solutions containing conjugates of different ratios; the TAS are presented as heat maps in Figure 4A-C. Briefly, three features could be observed: an initial ground state bleach centered at $350 \mathrm{~nm}$, a narrow excited state absorption centered at $375 \mathrm{~nm}$, and a broader excited state absorption centered at $490 \mathrm{~nm}$. These features started decaying at pump-probe time delays $(\Delta t)>1 \mathrm{ps}$, with their decay persisting to the maximum experimental $\Delta t=3 \mathrm{~ns}$ of the instrumental setup (see Figure S22 for different time delays chosen to highlight these features). To quantitatively analyze the dynamical processes observed, a global sequential fit $(\mathrm{A} \rightarrow \mathrm{B} \rightarrow \mathrm{C} \cdots)$ requiring up to five time constants was employed to extract the kinetics in the TAS data. The first two time constants for PYR-CP-PEG and PYR-CP-PEG/NTI-CP-PEG, and the first time constant for PYR-CP-PEG/NTI-CP-PEG/Cy3-CP-PEG were required to account for the instrument response (these likely include contributions from the solvent and the glass of the cuvette, thus the need in some circumstances for multiple components in the fit). The remaining three time constants (for each system) were very similar, implicating three transient species in the overall kinetic scheme with the following (approximate) time constants: 3 ps, $150 \mathrm{ps}$, and $\gg 3$ ns. The fitted data is presented as evolution-associated difference spectra (EADS) in Figure 4D-F. The addition of NTI-CP-PEG as the first FRET acceptor was evident in the TAS as the intensity of the broad excited state absorption centered at $490 \mathrm{~nm}$ faded and narrowed down (Figure 4B). Moreover, as evidenced in Figure 4E, the small decrease in intensity centered at $550 \mathrm{~nm}$ was also indicative of the energy transfer from PYR-CP-PEG to NTI-CP-PEG, which coincides with the stimulated emission observed in the TAS of NTI-CP-PEG on its own (Figure $\mathrm{S} 23 \mathrm{a}, \mathrm{e})$; the absence of a stimulated emission in Figure 4E could be due to masking from an excited state absorption. The addition of Cy3-CP-PEG as the second acceptor was 

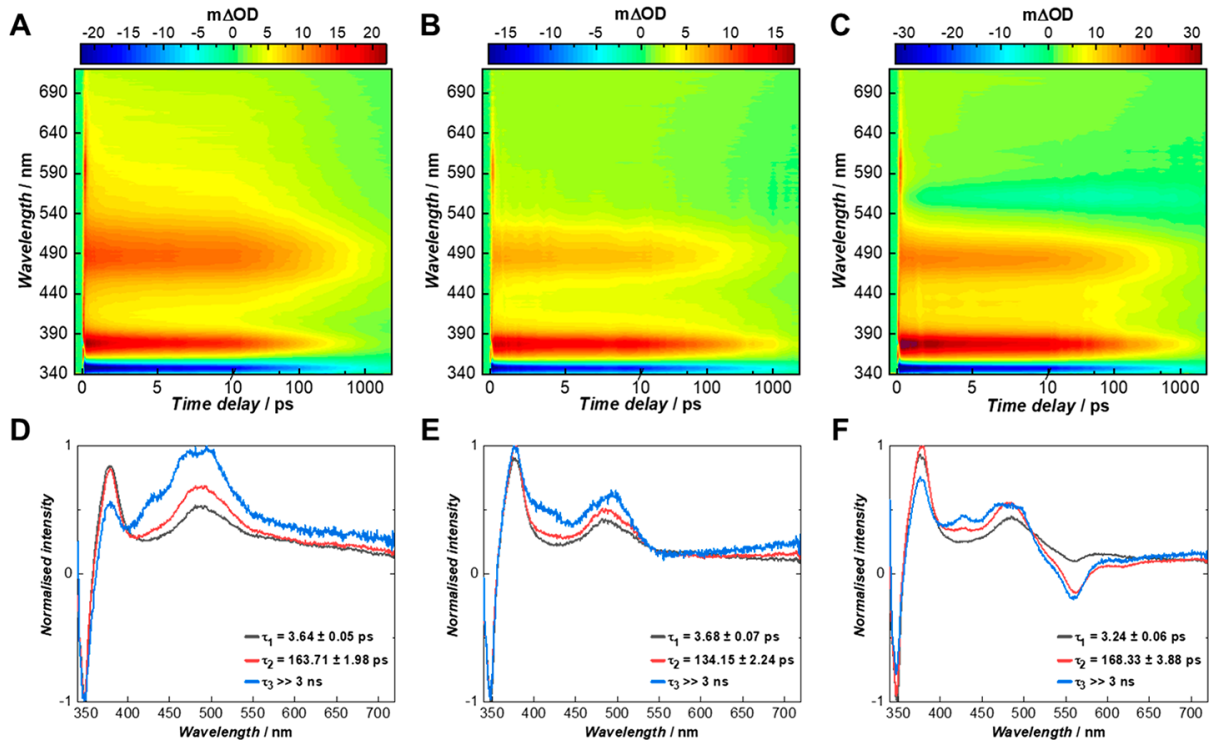

Figure 4. Transient electronic absorption spectroscopy study. (A-C) TAS of PYR-CP-PEG, PYR-CP-PEG/NTI-CP-PEG = 100/8, and PYR-CPPEG/NTI-CP-PEG/Cy3-CP-PEG = 100/8/2, respectively (pumped at an excitation wavelength of $335 \mathrm{~nm}$ ) represented as false color maps. (DF) Corresponding EADS for the above-mentioned solutions acquired from the global fit.

evidenced in Figure 4C,F through the formation of an intense stimulated emission feature centered at $560 \mathrm{~nm}$ (Figure $\mathrm{S} 23 \mathrm{~b}, \mathrm{f})$, confirming the energy being further transferred to Cy3-CP-PEG as the second acceptor is only negligibly excited when analyzed on its own at $335 \mathrm{~nm}$ (Figure S24b,e). This stimulated emission feature appears to grow in with increasing delay times, which could be indicative of a two-step sequential energy transfer process. In the meantime, the presence of the stimulated emission even at the earliest times might be explained by a direct energy transfer from PYR-CP-PEG to Cy3-CP-PEG.

Emission Color Tuning Utilizing the Three-Component System. The highly efficient FRET between PYR-CPPEG, NTI-CP-PEG, and Cy3-CP-PEG enables us to straightforwardly tune the emission color. ${ }^{50-52}$ To determine the color range that could be obtained from this threecomponent system, two solutions with relatively high acceptor/donor ratios (NTI-CP-PEG/PYR-CP-PEG = 1/1, Cy3-CPPEG/NTI-CP-PEG/PYR-CP-PEG = $1 / 1 / 1)$ were prepared, guaranteeing almost $100 \%$ FRET efficiency. Figure 5A shows the emission spectra of PYR-CP-PEG, NTI-CP-PEG/PYR-CP$\mathrm{PEG}=1 / 1$, and Cy3-CP-PEG/NTI-CP-PEG/PYR-CP-PEG $=$ $1 / 1 / 1$ excited at $335 \mathrm{~nm}$, which are then related to specific color coordinates in the CIE diagram. As expected, PYR-CPPEG showed blue color emission $(0.154,0.180)$, and the emission color turned into green $(0.353,0.593)$ with the addition of one equivalent NTI-CP-PEG. The emission color could be further adjusted to orange $(0.570,0.418)$ when adding another equivalent of Cy3-CP-PEG (Figure 5B). By adjusting different PYR-CP-PEG/NTI-CP-PEG/Cy3-CP-PEG ratios, self-assembled polymeric nanotubes with continuously tunable emission color within the triangular color gamut could be easily obtained (Figures 5C, S25, and S26). Furthermore, the high binding fidelity of the cyclic peptides endows the supramolecular polymeric nanotubes with unusually high stability. As shown in Figure S27, there was no significant variation of emission color when changing either concentration or temperature. Moreover, the emission color remained
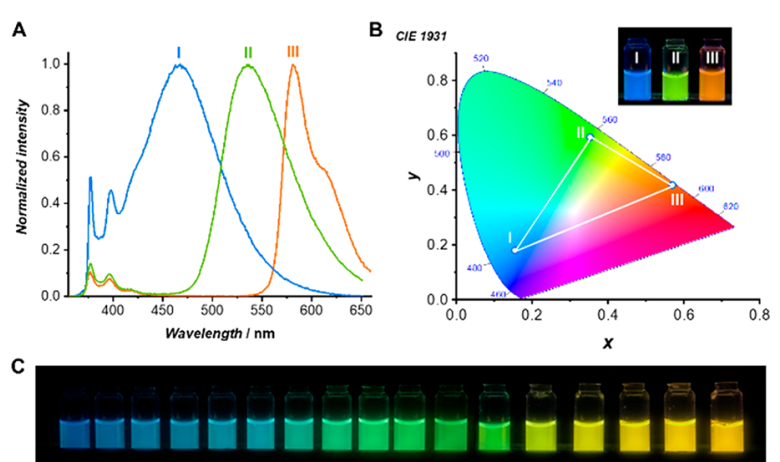

Figure 5. Emission color tuning. (A) Fluorescence spectra of PYRCP-PEG (I), PYR-CP-PEG/NTI-CP-PEG = 1/1 (II), and PYR-CPPEG/NTI-CP-PEG/Cy3-CP-PEG $=1 / 1 / 1$ (III) in water $\left(\lambda_{\text {ex }}=335\right.$ nm). (B) CIE 1931 diagram showing CIE coordinates of I, II, and III. White triangle: color area could be obtained by the three-component system. Insert: photograph of I, II, and III. (C) Photograph showing different emission colors at different PYR-CP-PEG/NTI-CP-PEG/ Cy3-CP-PEG ratios.

unchanged when integrating these supramolecular polymeric nanotubes into acrylamide hydrogels (Figure S28).

Notably, a white-light emission of $(0.321,0.336)$ in CIE coordinates could be obtained with the ratio of PYR-CP-PEG/ NTI-CP-PEG/Cy3-CP-PEG = 100/1/2 (Figure 6). Under this condition, $\Phi_{\mathrm{ET}}$ from PYR-CP-PEG to NTI-CP-PEG was calculated to be $37.0 \%$, while $\Phi_{\mathrm{ET}}$ from NTI-CP-PEG to Cy3CP-PEG was $67.9 \%$ (Figure S29). The fluorescence quantum yield was measured to be $29.9 \pm 3.1 \%$, close to the reported record value for white-light-emitting organic materials in water $(38 \%) .{ }^{31}$ Moreover, the white-emitting color could be maintained at a wide range of solution concentrations (Figure S30). Considering the high stability and high fluorescence quantum yield of the supramolecular polymeric nanotubes, this three-component system is believed to be suitable for applications as white-light-emitting materials. ${ }^{53,54}$ 


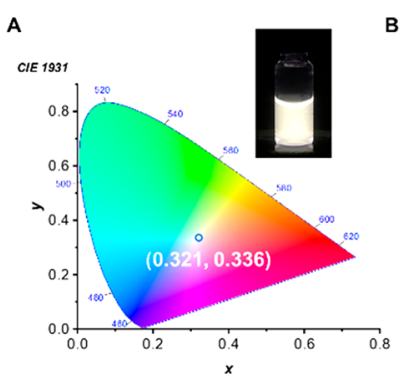

B

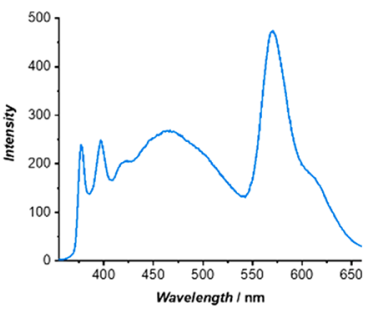

Figure 6. White-light emission. (A) CIE 1931 diagram showing white-light emitting coordinates. Insert: photograph of the white-light emission. (B) Fluorescence spectrum of the white-light emission. $([\mathrm{PYR}-\mathrm{CP}-\mathrm{PEG}]=50 \mu \mathrm{M},[\mathrm{NTI}-\mathrm{CP}-\mathrm{PEG}]=0.5 \mu \mathrm{M},[\mathrm{Cy} 3-\mathrm{CP}-$ PEG] $=1 \mu \mathrm{M}, \lambda_{\text {ex }}=335 \mathrm{~nm}$ ).

\section{CONCLUSIONS}

We have reported an artificial light-harvesting system in aqueous environment based on self-assembled peptide nanotubes constructed by cyclic peptide-polymer conjugates. By simply coassembling the three conjugates, PYR-CP-PEG, NTICP-PEG, and Cy3-CP-PEG, an efficient artificial lightharvesting system with two-step sequential FRET process was achieved. Through effective alignment of the donor and acceptor fluorophores thanks to the supramolecular peptide nanotubes, the ACQ effect which typically plagues these systems was significantly reduced, resulting in high fluorescence quantum yields $(\geq 30 \%)$ in aqueous media. The emission color could be continuously tuned from blue to green and finally to orange by adjusting the ratio of the three conjugates. Remarkably, pure white light with a fluorescence quantum yield as high as $29.9 \%$ is also accessible. Moreover, this system shows good stability, which could preserve its performance at low concentration $(<1 \mu \mathrm{M})$ and high temperature $\left(80^{\circ} \mathrm{C}\right)$. This supramolecular peptide nanotubebased system is expected to serve as an efficient artificial lightharvesting system with potential light-emitting applications. Moreover, to better mimic the natural photosynthesis process, acceptor fluorophores with photocatalytic activities could be used to conduct photochemical reactions, thus making full use of the harvested energy.

\section{ASSOCIATED CONTENT}

\section{SI Supporting Information}

The Supporting Information is available free of charge at https://pubs.acs.org/doi/10.1021/jacs.0c11060.

Materials and instruments, details about synthetic procedures and characterization, and supplemental figures $(\mathrm{PDF})$

\section{AUTHOR INFORMATION}

\section{Corresponding Author}

Sébastien Perrier - Department of Chemistry, University of Warwick, Coventry CV4 7AL, United Kingdom; Warwick Medical School, University of Warwick, Coventry CV4 7AL, United Kingdom; Faculty of Pharmacy and Pharmaceutical Sciences, Monash University, Parkville, Victoria 3052, Australia; ○ orcid.org/0000-0001-5055-9046;

Email: s.perrier@warwick.ac.uk

\section{Authors}

Qiao Song - Department of Chemistry, University of Warwick, Coventry CV4 7AL, United Kingdom

Sofia Goia - Department of Chemistry, University of Warwick, Coventry CV4 7AL, United Kingdom; Molecular Analytical Science Centre for Doctoral Training, Senate House, University of Warwick, Coventry CV4 7AL, United Kingdom

Jie Yang - Department of Chemistry, University of Warwick, Coventry CV4 7AL, United Kingdom; 이이.org/00000001-9970-7505

Stephen C. L. Hall - Department of Chemistry, University of Warwick, Coventry CV4 7AL, United Kingdom; - orcid.org/0000-0003-0753-5123

Michael Staniforth - Department of Chemistry, University of Warwick, Coventry CV4 7AL, United Kingdom

Vasilios G. Stavros - Department of Chemistry, University of Warwick, Coventry CV4 7AL, United Kingdom; (1) orcid.org/0000-0002-6828-958X

Complete contact information is available at: https://pubs.acs.org/10.1021/jacs.0c11060

\section{Author Contributions}

The manuscript was written through contributions of all authors.

\section{Notes}

The authors declare no competing financial interest.

\section{ACKNOWLEDGMENTS}

The Royal Society Wolfson Merit Award (WM130055; S.P.), Monash-Warwick Alliance (S.C.L.H.; S.P.), the European Research Council (TUSUPO 647106; Q.S.; S.P.), the Royal Society and Leverhulme Trust (LT150052; V.G.S.), and the Marie Sklodowska-Curie action (TSPBNTM; J.Y.) are acknowledged for financial support. The authors thank Dr. Robert Dalgliesh (ISIS, Oxford, U.K.), Dr Judith Houston (European Spallation Source, Lund, Sweden), Ms. Maria Kariuki, and Ms. Sophie Hill for assistance with SANS experiment. We also acknowledge the STFC for the allocation of beam time at ISIS (RB1920524). The authors also thank the Warwick Centre for Ultrafast Spectroscopy (https://warwick. ac.uk/fac/sci/wcus). S.G. acknowledges the EPSRC for a PhD studentship through the EPSRC Centre for Doctoral Training in Molecular Analytical Science (EP/L015307/1).

\section{REFERENCES}

(1) Croce, R.; van Amerongen, H. Natural strategies for photosynthetic light harvesting. Nat. Chem. Biol. 2014, 10 (7), 492-501.

(2) Scholes, G. D.; Fleming, G. R.; Olaya-Castro, A.; van Grondelle, R. Lessons from nature about solar light harvesting. Nat. Chem. 2011, 3 (10), 763-774.

(3) Ahn, T. K.; Avenson, T. J.; Ballottari, M.; Cheng, Y.-C.; Niyogi, K. K.; Bassi, R.; Fleming, G. R. Architecture of a charge-transfer state regulating light harvesting in a plant antenna protein. Science 2008, 320 (5877), 794.

(4) Wei, X.; Su, X.; Cao, P.; Liu, X.; Chang, W.; Li, M.; Zhang, X.; Liu, Z. Structure of spinach photosystem II-LHCII supercomplex at 3.2 Å resolution. Nature 2016, 534 (7605), 69-74.

(5) Son, M.; Pinnola, A.; Bassi, R.; Schlau-Cohen, G. S. The electronic structure of lutein 2 is optimized for light harvesting in plants. Chem. 2019, 5 (3), 575-584.

(6) Mirkovic, T.; Ostroumov, E. E.; Anna, J. M.; van Grondelle, R.; Govindjee; Scholes, G. D. Light absorption and energy transfer in the antenna complexes of photosynthetic organisms. Chem. Rev. 2017, 117 (2), 249-293. 
(7) Peng, H.-Q.; Niu, L.-Y.; Chen, Y.-Z.; Wu, L.-Z.; Tung, C.-H.; Yang, Q.-Z. Biological applications of supramolecular assemblies designed for excitation energy transfer. Chem. Rev. 2015, 115 (15), $7502-7542$.

(8) Zou, Q.; Liu, K.; Abbas, M.; Yan, X. Peptide-modulated selfassembly of chromophores toward biomimetic light-harvesting nanoarchitectonics. Adv. Mater. 2016, 28 (6), 1031-1043.

(9) Schulze, M.; Kunz, V.; Frischmann, P. D.; Würthner, F. A supramolecular ruthenium macrocycle with high catalytic activity for water oxidation that mechanistically mimics photosystem II. Nat. Chem. 2016, 8 (6), 576-583.

(10) Chen, P.-Z.; Weng, Y.-X.; Niu, L.-Y.; Chen, Y.-Z.; Wu, L.-Z.; Tung, C.-H.; Yang, Q.-Z. Light-harvesting systems based on organic nanocrystals to mimic chlorosomes. Angew. Chem., Int. Ed. 2016, 55 (8), 2759-2763.

(11) Hahn, U.; Gorka, M.; Vögtle, F.; Vicinelli, V.; Ceroni, P.; Maestri, M.; Balzani, V. Light-harvesting dendrimers: efficient intraand intermolecular energy-transfer processes in a species containing 65 chromophoric groups of four different types. Angew. Chem., Int. Ed. 2002, 41 (19), 3595-3598.

(12) Arrigo, A.; Puntoriero, F.; La Ganga, G.; Campagna, S.; Burian, M.; Bernstorff, S.; Amenitsch, H. Aggregation-induced energy transfer in a decanuclear $\mathrm{Os}(\mathrm{II}) / \mathrm{Ru}(\mathrm{II})$ polypyridine light-harvesting antenna dendrimer. Chem. 2017, 3 (3), 494-508.

(13) Zhang, D.; Liu, Y.; Fan, Y.; Yu, C.; Zheng, Y.; Jin, H.; Fu, L.; Zhou, Y.; Yan, D. Hierarchical self-assembly of a dandelion-like supramolecular polymer into nanotubes for use as highly efficient aqueous light-harvesting systems. Adv. Funct. Mater. 2016, 26 (42), $7652-7661$

(14) Sarkar, A.; Behera, T.; Sasmal, R.; Capelli, R.; Empereur-mot, C.; Mahato, J.; Agasti, S. S.; Pavan, G. M.; Chowdhury, A.; George, S. J. Cooperative supramolecular block copolymerization for the synthesis of functional axial organic heterostructures. J. Am. Chem. Soc. 2020, 142 (26), 11528-11539.

(15) Kim, H.-J.; Nandajan, P. C.; Gierschner, J.; Park, S. Y. Lightharvesting fluorescent supramolecular block copolymers based on cyanostilbene derivatives and cucurbit[8]urils in aqueous solution. Adv. Funct. Mater. 2018, 28 (4), 1705141.

(16) Zhang, X.; Chen, Z.-K.; Loh, K. P. Coordination-assisted assembly of 1-D nanostructured light-harvesting antenna. J. Am. Chem. Soc. 2009, 131 (21), 7210-7211.

(17) Ji, L.; Sang, Y.; Ouyang, G.; Yang, D.; Duan, P.; Jiang, Y.; Liu, M. Cooperative chirality and sequential energy transfer in a supramolecular light-harvesting nanotube. Angew. Chem., Int. Ed. 2019, 58 (3), 844-848.

(18) Xu, L.; Wang, Z.; Wang, R.; Wang, L.; He, X.; Jiang, H.; Tang, H.; Cao, D.; Tang, B. Z. A conjugated polymeric supramolecular network with aggregation-induced emission enhancement: an efficient light-harvesting system with an ultrahigh antenna effect. Angew. Chem., Int. Ed. 2020, 59 (25), 9908-9913.

(19) Peng, H.-Q.; Chen, Y.-Z.; Zhao, Y.; Yang, Q.-Z.; Wu, L.-Z.; Tung, C.-H.; Zhang, L.-P.; Tong, Q.-X. Artificial light-harvesting system based on multifunctional surface-cross-linked micelles. Angew. Chem., Int. Ed. 2012, 51 (9), 2088-2092.

(20) Li, J.-J.; Chen, Y.; Yu, J.; Cheng, N.; Liu, Y. A supramolecular artificial light-harvesting system with an ultrahigh antenna effect. Adv. Mater. 2017, 29 (30), 1701905.

(21) Xu, Z.; Peng, S.; Wang, Y.-Y.; Zhang, J.-K.; Lazar, A. I.; Guo, D.-S. Broad-spectrum tunable photoluminescent nanomaterials constructed from a modular light-harvesting platform based on macrocyclic amphiphiles. Adv. Mater. 2016, 28 (35), 7666-7671.

(22) Guo, S.; Song, Y.; He, Y.; Hu, X.-Y.; Wang, L. Highly efficient artificial light-harvesting systems constructed in aqueous solution based on supramolecular self-assembly. Angew. Chem., Int. Ed. 2018, 57 (12), 3163-3167.

(23) Hao, M.; Sun, G.; Zuo, M.; Xu, Z.; Chen, Y.; Hu, X.-Y.; Wang, L. A supramolecular artificial light-harvesting system with two-step sequential energy transfer for photochemical catalysis. Angew. Chem., Int. Ed. 2020, 59 (25), 10095-10100.
(24) Channon, K. J.; Devlin, G. L.; MacPhee, C. E. Efficient energy transfer within self-assembling peptide fibers: a route to lightharvesting nanomaterials. J. Am. Chem. Soc. 2009, 131 (35), 1252012521.

(25) Dutta, P. K.; Levenberg, S.; Loskutov, A.; Jun, D.; Saer, R.; Beatty, J. T.; Lin, S.; Liu, Y.; Woodbury, N. W.; Yan, H. A DNAdirected light-harvesting/reaction center system. J. Am. Chem. Soc. 2014, 136 (47), 16618-16625.

(26) Dutta, P. K.; Varghese, R.; Nangreave, J.; Lin, S.; Yan, H.; Liu, Y. DNA-directed artificial light-harvesting antenna. J. Am. Chem. Soc. 2011, 133 (31), 11985-11993.

(27) Hirayama, S.; Oohora, K.; Uchihashi, T.; Hayashi, T. Thermoresponsive micellar assembly constructed from a hexameric hemoprotein modified with poly(N-isopropylacrylamide) toward an artificial light-harvesting system. J. Am. Chem. Soc. 2020, 142 (4), $1822-1831$

(28) Li, J.; Wang, J.; Li, H.; Song, N.; Wang, D.; Tang, B. Z. Supramolecular materials based on AIE luminogens (AIEgens): construction and applications. Chem. Soc. Rev. 2020, 49 (4), 11441172.

(29) Tang, B. Z.; Zhao, Z.; Zhang, H.; Lam, J. W. Y. Aggregationinduced emission: new vistas at aggregate level. Angew. Chem., Int. Ed. 2020, 59 (25), 9888-9907.

(30) Hong, Y.; Lam, J. W. Y.; Tang, B. Z. Aggregation-induced emission. Chem. Soc. Rev. 2011, 40 (11), 5361-5388.

(31) Li, C.; Zhang, J.; Zhang, S.; Zhao, Y. Efficient light-harvesting systems with tunable emission through controlled precipitation in confined nanospace. Angew. Chem., Int. Ed. 2019, 58 (6), 1643-1647.

(32) Li, Y.; Dong, Y.; Cheng, L.; Qin, C.; Nian, H.; Zhang, H.; Yu, Y.; Cao, L. Aggregation-induced emission and light-harvesting function of tetraphenylethene-based tetracationic dicyclophane. $J$. Am. Chem. Soc. 2019, 141 (21), 8412-8415.

(33) Acharyya, K.; Bhattacharyya, S.; Sepehrpour, H.; Chakraborty, S.; Lu, S.; Shi, B.; Li, X.; Mukherjee, P. S.; Stang, P. J. Self-assembled fluorescent $\mathrm{Pt}(\mathrm{II})$ metallacycles as artificial light-harvesting systems. J. Am. Chem. Soc. 2019, 141 (37), 14565-14569.

(34) Li, Z.; Han, Y.; Wang, F. Compartmentalization-induced phosphorescent emission enhancement and triplet energy transfer in aqueous medium. Nat. Commun. 2019, 10 (1), 3735.

(35) Li, J.-J.; Zhang, H.-Y.; Dai, X.-Y.; Liu, Z.-X.; Liu, Y. A highly efficient light-harvesting system with sequential energy transfer based on a multicharged supramolecular assembly. Chem. Commun. 2020, 56 (44), 5949-5952.

(36) Shimizu, T.; Ding, W.; Kameta, N. Soft-matter nanotubes: a platform for diverse functions and applications. Chem. Rev. 2020, 120 (4), 2347-2407.

(37) Couet, J.; Samuel, J. D. J. S.; Kopyshev, A.; Santer, S.; Biesalski, M. Peptide-polymer hybrid nanotubes. Angew. Chem., Int. Ed. 2005, 44 (21), 3297-3301.

(38) ten Cate, M. G. J.; Severin, N.; Börner, H. G. Self-assembling peptide-polymer conjugates comprising (D-alt-L)-cyclopeptides as aggregator domains. Macromolecules 2006, 39 (23), 7831-7838.

(39) Chapman, R.; Jolliffe, K. A.; Perrier, S. Modular design for the controlled production of polymeric nanotubes from polymer/peptide conjugates. Polym. Chem. 2011, 2 (9), 1956-1963.

(40) Hourani, R.; Zhang, C.; van der Weegen, R.; Ruiz, L.; Li, C.; Keten, S.; Helms, B. A.; Xu, T. Processable cyclic peptide nanotubes with tunable interiors. J. Am. Chem. Soc. 2011, 133 (39), 1529615299.

(41) Chapman, R.; Danial, M.; Koh, M. L.; Jolliffe, K. A.; Perrier, S. Design and properties of functional nanotubes from the self-assembly of cyclic peptide templates. Chem. Soc. Rev. 2012, 41 (18), 60236041.

(42) Brea, R. J.; Reiriz, C.; Granja, J. R. Towards functional bionanomaterials based on self-assembling cyclic peptide nanotubes. Chem. Soc. Rev. 2010, 39 (5), 1448-1456.

(43) Ghadiri, M. R.; Granja, J. R.; Milligan, R. A.; McRee, D. E.; Khazanovich, N. Self-assembling organic nanotubes based on a cyclic peptide architecture. Nature 1993, 366 (6453), 324-327. 
(44) Rho, J. Y.; Brendel, J. C.; MacFarlane, L. R.; Mansfield, E. D. H.; Peltier, R.; Rogers, S.; Hartlieb, M.; Perrier, S. Probing the dynamic nature of self-assembling cyclic peptide-polymer nanotubes in solution and in mammalian cells. Adv. Funct. Mater. 2018, 28 (24), 1704569.

(45) Rho, J. Y.; Cox, H.; Mansfield, E. D. H.; Ellacott, S. H.; Peltier, R.; Brendel, J. C.; Hartlieb, M.; Waigh, T. A.; Perrier, S. Dual selfassembly of supramolecular peptide nanotubes to provide stabilisation in water. Nat. Commun. 2019, 10 (1), 4708.

(46) Yang, J.; Song, J.-I.; Song, Q.; Rho, J. Y.; Mansfield, E. D. H.; Hall, S. C. L.; Sambrook, M.; Huang, F.; Perrier, S. Hierarchical selfassembled photo-responsive tubisomes from a cyclic peptide-bridged amphiphilic block copolymer. Angew. Chem., Int. Ed. 2020, 59 (23), $8860-8863$.

(47) Bressler, I.; Kohlbrecher, J.; Thunemann, A. F. SASfit: a tool for small-angle scattering data analysis using a library of analytical expressions. J. Appl. Crystallogr. 2015, 48 (5), 1587-1598.

(48) Clegg, R. M. Fluorescence resonance energy transfer. Curr. Opin. Biotechnol. 1995, 6 (1), 103-110.

(49) Broussard, J. A.; Rappaz, B.; Webb, D. J.; Brown, C. M. Fluorescence resonance energy transfer microscopy as demonstrated by measuring the activation of the serine/threonine kinase Akt. Nat. Protoc. 2013, 8 (2), 265-281.

(50) Wu, H.; Chen, Y.; Dai, X.; Li, P.; Stoddart, J. F.; Liu, Y. In situ photoconversion of multicolor luminescence and pure white light emission based on carbon dot-supported supramolecular assembly. $J$. Am. Chem. Soc. 2019, 141 (16), 6583-6591.

(51) Zhang, M.; Yin, S.; Zhang, J.; Zhou, Z.; Saha, M. L.; Lu, C.; Stang, P. J. Metallacycle-cored supramolecular assemblies with tunable fluorescence including white-light emission. Proc. Natl. Acad. Sci. U. S. A. 2017, 114 (12), 3044.

(52) Zuo, M.; Qian, W.; Li, T.; Hu, X.-Y.; Jiang, J.; Wang, L. Fullcolor tunable fluorescent and chemiluminescent supramolecular nanoparticles for anti-counterfeiting inks. ACS Appl. Mater. Interfaces 2018, 10 (45), 39214-39221.

(53) Li, D.; Wang, J.; Ma, X. White-light-emitting materials constructed from supramolecular approaches. Adv. Opt. Mater. 2018, 6 (20), 1800273.

(54) Praveen, V. K.; Ranjith, C.; Armaroli, N. White-light-emitting supramolecular gels. Angew. Chem., Int. Ed. 2014, 53 (2), 365-368. 